\title{
Enhancement of TRAIL cytotoxicity by AG-490 in human ALL cells is characterized by downregulation of cIAP-1 and cIAP-2 through inhibition of Jak2/Stat3
}

\author{
Paola Lanuti ${ }^{1,2, *}$, Valeria Bertagnolo ${ }^{3, *}$, Laura Pierdomenico ${ }^{1,2}$, Adriana Bascelli ${ }^{1,2}$, Eugenio Santavenere ${ }^{1}$, \\ Lapo Alinari ${ }^{4,5}$, Silvano Capitani ${ }^{3}$, Sebastiano Miscia ${ }^{1,2}$, Marco Marchisio ${ }^{1,2}$ \\ ${ }^{I}$ Cell Signalling Unit, Department of Biomorphology, University “G. d'Annunzio” of Chieti-Pescara, Via dei Vestini 13, 66013 \\ Chieti, Italy; ${ }^{2}$ Center for Ageing Sciences (Ce.S.I.), "Università G.d'Annunzio” Foundation, Chieti, Italy; ${ }^{3}$ Cell Signalling Unit, \\ Department of Morphology and Embryology, University of Ferrara, Ferrara, Italy; ${ }^{4}$ Institute of Hematology and Medical Oncol- \\ ogy "L.\&A. Seragnoli", University of Bologna, Bologna, Italy; ${ }^{5}$ Division of Haematology-Oncology, Department of Internal Medi- \\ cine, The Ohio State University, Columbus, OH, USA
}

The ability of death-inducing tumor necrosis factor-related apoptosis-inducing ligand (TRAIL) to selectively kill a variety of cancer cells has been largely described, but one of the major concerns with the treatment is the occurrence of drug resistance and possible toxic side effects. Here, we report that TRAIL induces apoptosis in Jurkat and SUPT1 $T$ cell lines and in human T-ALL blasts but not in healthy subject-derived peripheral blood mononuclear cells. In parallel, the treatment with TRAIL and Tyrphostin (AG-490), a selective Janus kinase 2 inhibitor, produces an evident enhancement of cytotoxicity, characterized by a significant inhibition of Stat 3 phosphorylation compared to controls or to TRAIL alone-treated samples, and associated with a dramatic decrease of both cIAP-1 and cIAP-2 mRNA levels. Downregulation of cIAP-1 and cIAP-2 by specific small interference RNAs significantly amplifies TRAIL-reduced cytotoxicity. All together, these findings strongly indicate that cIAP-1 and cIAP-2 downregulation is a fundamental step in the signaling pathways mediating the combinatorial effect of TRAIL and AG-490 on T cell leukemia. These findings may help to open new routes for the development of less toxic pharmacological strategies in the treatment of patients affected by TRAIL-sensitive leukemias.

Keywords: ALL, AG-490, TRAIL, Stat3, IAPs, apoptosis

Cell Research (2009) 19:1079-1089. doi: 10.1038/cr.2009.80; published online 30 June 2009

\section{Introduction}

Acute lymphoblastic leukemia (ALL) is a malignant disorder originating from a single B- or T-lymphocyte progenitor. Leukemic cell proliferation and accumulation give rise to the suppression of healthy haematopoiesis and involve different organs including the liver, lymph nodes, thymus, spleen, gonads and meninges. Recent reports indicate that in children and adolescents with ALL,

\footnotetext{
*These two authors contributed equally to this work. Correspondence: Sebastiano Miscia

Tel: +39 08713555304; Fax: +390871574361

E-mail: s.miscia@unich.it

Received 15 August 2008; revised 22 February 2009; accepted 2 April 2009; published online 30 June 2009
}

an overall cure rate of $90 \%$ may be achieved [1] but that many patients still develop serious acute and late complications owing to the side effects of treatments. Cure rates of T-ALL in adults are considerably lower than in the younger population and relapsed T-ALL continues to confer a sad prognosis as evidenced by the 5 -year survival rates in adults ( $32 \%$ to $46 \%$ ) [2]. The search for more effective and safer anti-leukemia therapies continues to identify agents and combinations of agents with specific activity against the disease with the aim to devise new treatment approaches to improve both the cure rate and the life quality of T-ALL patients.

One promising approach that is of interest in the treatment of human $\mathrm{T}$ leukemia is the use of death-inducing tumor necrosis factor (TNF)-related apoptosis-inducing ligand (TRAIL). TRAIL has attracted interest because of 
its selective apoptotic activity on a number of tumor cell types without displaying toxicity for normal cells $[3,4]$. TRAIL interacts with a complex receptor system, which includes at least two agonistic receptors (death receptors) and three antagonistic receptors. TRAIL-R1 and TRAILR2 (agonistic receptors) contain a cytoplasmic death domain, eliciting apoptotic signals upon TRAIL binding. TRAIL-R3, TRAIL-R4 and Osteoprogerin (antagonistic receptors) do not display a functional death domain and may act as decoy receptors [5], possibly protecting normal cells and TRAIL-resistant tumor cells from apoptosis. The regulation and the function of TRAIL are complex, as results from different studies suggest no relationship between TRAIL sensitivity and the expression of antagonistic receptors [6]. Therefore, the mechanism related to the tumor-selective activity of TRAIL requires further investigations. The signaling system responsible for the apoptotic cell death is activated by a TRAILdependent clustering of TRAIL-R1 and TRAIL-R2, which induces in turn the activation of the initiator and the effector procaspases, resulting in cell death induction. TRAIL resistance of certain tumors and the scarce knowledge about the consequence of TRAIL on normal cells, present a potential concern about this type of therapy. In this context, the combination of TRAIL with other chemotherapeutic drugs or with ionizing radiations has already been attempted in order to obtain a synergistic effect against tumor cells [7, 8]. Tyrphostin (AG-490) has been reported to be a molecule with promising clinical potential as an immunotherapeutic drug due to its inhibitory effects on T cell-signaling pathways [9-12]. AG-490 is a tyrosine kinase inhibitor able to selectively block Janus kinase 2 (Jak2) activity, suppressing, in turn, Jak/ Stat (signal transducers and activators of transcription) signaling. The Jak/Stat cascade modulates the proliferation of different cancer cell lines [13, 14], including adult $\mathrm{T}$ cell leukemia/lymphoma cells [15]. Studies in animals have indicated that AG-490 administration prolonged rat allograft survival and inhibited IL-2-induced activation and translocation of $S t a t 5 \mathrm{a} / \mathrm{b}$ into the nucleus of lymphocytes infiltrating the irradiated spleen allotransplant [16]. The potential clinical relevance of AG-490 has been concluded from the observation that systemic administration of AG-490 suppresses the growth of acute lymphoid leukemia cell lines injected into SCID mice [17]. Very recently, it has been demonstrated that AG-490 efficiently blocks even Jak3 activity in T cells $[12,18]$. Jak proteins are cytosolic tyrosine kinases critical for mediating proliferation, differentiation and survival signals $[19,20]$. Recent findings revealed that Jak2/Stat3 activity confers resistance to apoptosis to lymphoid tumor cells [21-23] by means of upregulation of inhibitor of apoptosis pro- teins (IAPs) [24] and Fas-associated death-domain-like interleukin-1 $\beta$-converting enzyme (FLICE) inhibitory protein (cFLIP) [25]. IAPs represent a family of proteins expressed in various cell types, including haematopoietic cells. These proteins have been shown to suppress apoptosis by inhibiting active caspase-3, caspase- 7 and procaspase-9 [26-28]. Since we have previously reported a synergistic influence of AG-490 on TNF-mediated apoptosis, in this study we investigated the biological effects of the combination of AG-490 and scalar doses of TRAIL on human lymphoblastic leukemia cells and examined the associated signaling events.

\section{Results}

Cytotoxic effect of TRAIL alone or in combination with AG-490 in Jurkat and SUPT1 T cell lines

It has been shown that $50 \mu \mathrm{M}$ AG-490 blocks hyperactive forms of Jak2 in ALL [17]. We evaluated the cytotoxic effect of TRAIL alone or in combination with AG$490(50 \mu \mathrm{M})$ by using different approaches: (a) 3-(4,5dimethylthiazol-2-yl)2,5-diphenyltetrazolium bromide (MTT) assay, (b) count of viable cells by trypan blue exclusion assay and (c) flow cytometric analysis of apoptosis by simultaneously staining the cells for phosphatidylserine surface exposure and propidium iodide (PI) incorporation. Results from MTT assay indicated that after the addition of TRAIL alone, at different concentrations (ranging from 5 to $300 \mathrm{ng} / \mathrm{ml}$ ), Jurkat cells showed a distinct pattern of responses: a modest sensitivity to apoptosis in presence of 5 or $10 \mathrm{ng} / \mathrm{ml}$ TRAIL, and an increased sensitivity within the range of $25-300 \mathrm{ng} / \mathrm{ml}$ but without any significant difference among the concentrations included in this range (Figure 1A and 1B). We then investigated the cytotoxicity when AG-490 $(50 \mu \mathrm{M})$ was combined with different concentrations of TRAIL. As summarized in Figure 1A and 1B, by combining both of the compounds together, a dramatic increase of the cytotoxic effects with respect to the use of TRAIL alone was found. Maximal cytotoxic effects were achieved when AG-490 $(50 \mu \mathrm{M})$ was combined with TRAIL used at concentrations ranging between 25 and $300 \mathrm{ng} / \mathrm{ml}$. Therefore, with the aim of using a minimum dose of TRAIL without impairing its specific apoptotic effects, we used $25 \mathrm{ng} / \mathrm{ml}$ TRAIL for further experiments, either alone or in combination with AG-490. Similar results have been obtained with SUPT1 cells. Trypan blue exclusion test and flow cytometry analysis of apoptosis, performed in Jurkat (Figure 1C and 1D) and SUPT1 (Figure $1 \mathrm{E}$ and $1 \mathrm{~F}$ ) cell lines, largely confirmed the data reported in Figure 1A and 1B. 


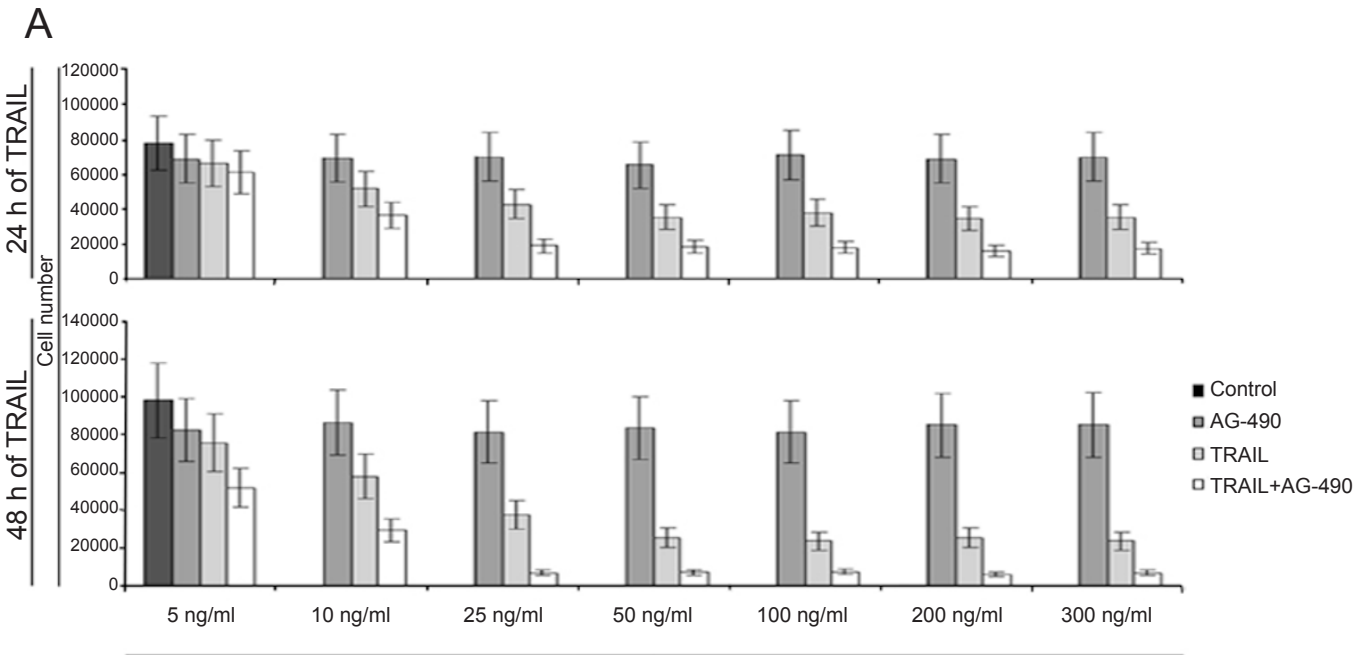

TRAIL concentration
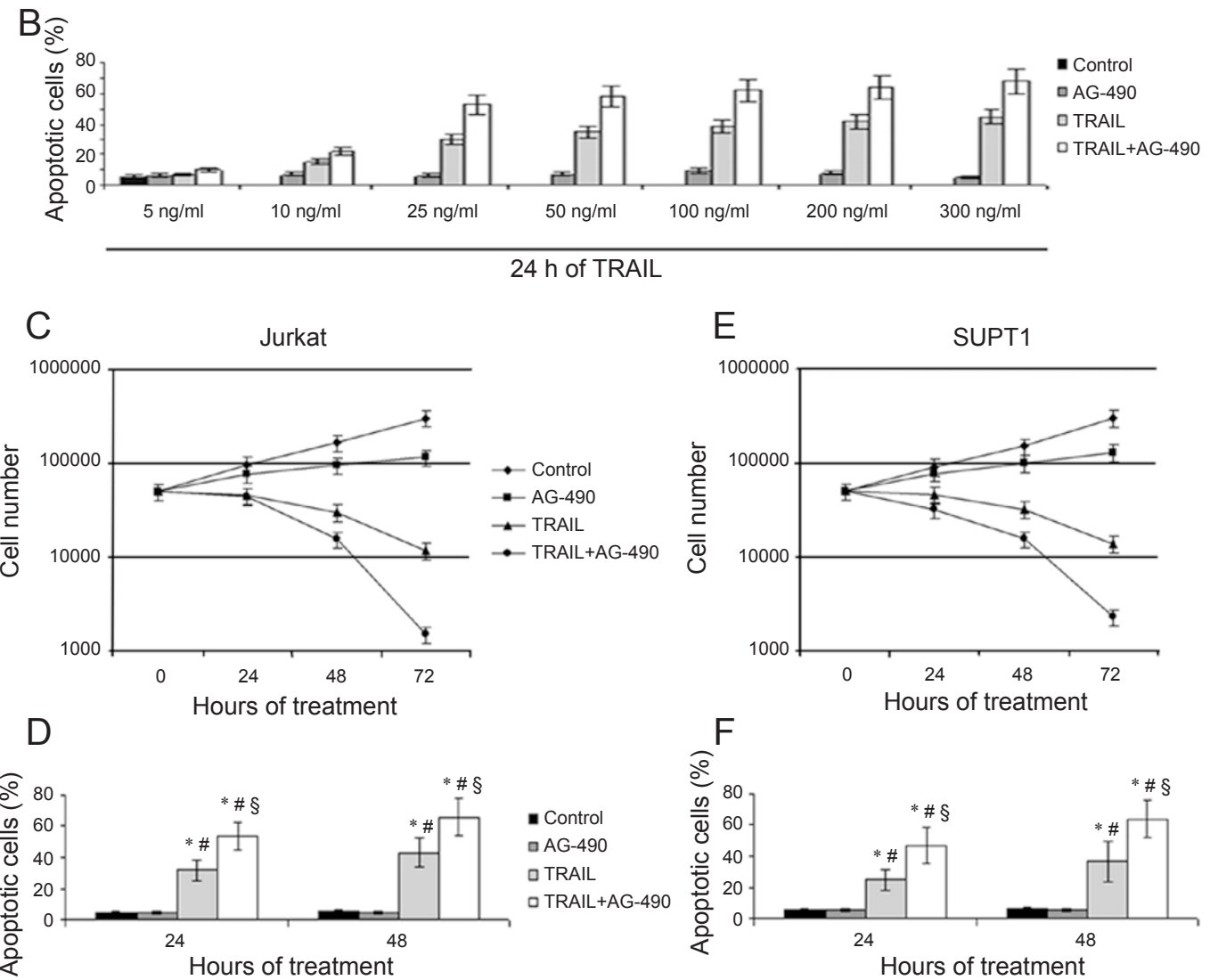

Figure 1 Susceptibility of Jurkat and SUPT1 cells to the cytotoxic activity of TRAIL or AG-490 used alone or in combination. Cytotoxicity evaluation (MTT assay) (A) and flow cytometry analysis of apoptosis (B) in Jurkat cells treated with AG-490 (50 $\mu \mathrm{M})$ or with different concentrations of TRAIL alone, or with both of the agonists combined together. Data are the mean of three separate experiments \pm SD. Evaluation of Jurkat (C) and SUPT1 (E) cell growth after the treatment with AG-490 (50 $\mu \mathrm{M})$, TRAIL $(25 \mathrm{ng} / \mathrm{ml})$ or of both of the agonists combined together. Cell number over 3 days of culture was evaluated by trypan blue exclusion test. Data are the mean of three separate experiments \pm SD. Flow cytometry analysis of apoptosis in Jurkat (D) and SUPT1 (F) cells treated for 24 or $48 \mathrm{~h}$ by AG-490 $(50 \mu \mathrm{M})$ or TRAIL $(25 \mathrm{ng} / \mathrm{ml})$ or both of the agonists combined together. Apoptosis was assessed by evaluating phosphatydylserine surface expression. Data are the mean of three separate experiments $\pm \mathrm{SD}$; * \# $(P<0.01)$ indicates statistically significant values with respect to control samples; $\S(P<0.01)$ indicates statistically significant values with respect to samples treated by TRAIL alone. In this figure, all samples treated with AG-490 were pre-treated for $12 \mathrm{~h}$. 
Combination of TRAIL and AG-490 increases the induction of apoptosis with respect to TRAIL alone in Jurkat cells and in human T-ALL primary blasts but not in healthy subject-derived PBMCs

The degree of apoptosis following independent treatments with TRAIL, AG-490 or both compounds together, was investigated by flow cytometric analyses in Jurkat cells and T-ALL-derived blasts, compared to healthy donor-derived peripheral blood mononuclear cells (PBMCs). The results indicated that the treatment with TRAIL alone produced a distinct degree of apoptosis in both Jurkat cells and in T-ALL-derived blasts but not in healthy donor-derived PBMCs (Figure 2A). No significant effect was noticed following AG-490 treatment of Jurkat cells, T-ALL blasts and healthy PBMCs. Of note, compared to the treatment with TRAIL alone, the combination of the two compounds gave rise to a distinct increase of the number of apoptotic events, both in Jurkat cells and T-ALL-derived blasts, without affecting healthy PBMCs. In order to investigate to what extent such synergic activity was reproducible, blasts derived from five T-ALL patients were analyzed by flow cytometry. Results, summarized in Figure 2B, showed that the combination of the two compounds produced higher cytotoxicity than TRAIL alone even though the amplitude of increase varied among the patients.

Surface expression of TRAIL receptors is not influenced by the treatment with $A G-490$

It has been previously demonstrated that toxic agents are able to modulate TRAIL receptors in different cell types [4] through different molecular pathways. Therefore, we investigated whether AG-490 treatment was able to modify the surface expression of the different TRAIL receptors on Jurkat and SUPT1 cells. Flow cytometric analysis indicated that, with respect to controls, AG-490 did not modulate the surface expression pattern of TRAIL receptors, demonstrating that the increase of the cytotoxic effect, following the addition of AG-490 to TRAIL, is not related to changes of the surface expression of TRAIL receptors (Supplementary information, Figure S1).

AG-490-dependent P-Stat3 downregulation is associated with the reduction of CIAP-1 and CIAP-2 $M R N A$ and protein levels

AG-490 is a well-known Jak2 inhibitor, able to effectively block Stat 3 activation in different cancer cell lines [13]. Activation of Stat3, on the other hand, has been associated with increased expression of different apoptosis inhibitors [23]. We therefore sought to determine whether AG-490 was effective in inhibiting Stat3 phosphorylation
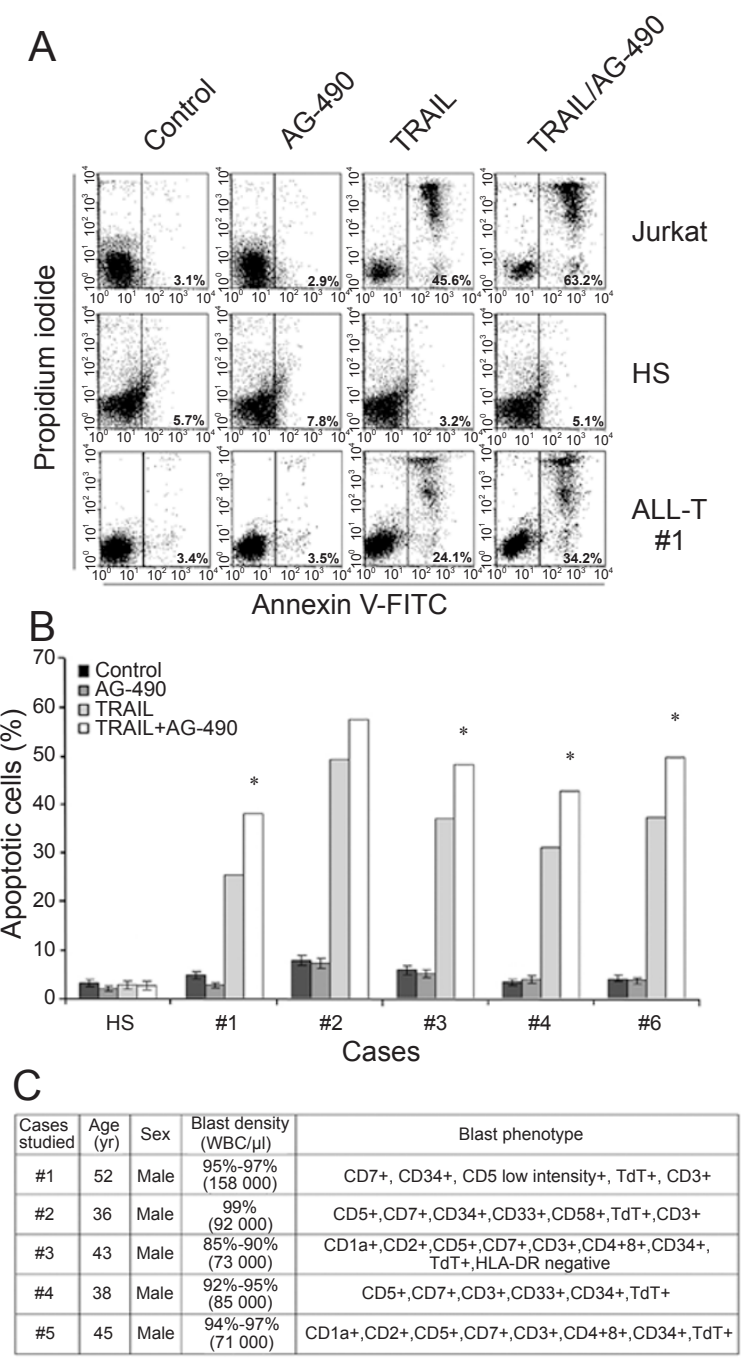

Figure 2 Flow cytometry analysis of apoptosis in Jurkat cells, PBMCs derived from healthy subjects and human T-ALL blasts after the treatment with AG-490, TRAIL or with both agonists combined together. (A) Flow cytometry analysis of apoptosis in Jurkat cells, in PBMCs from healthy subjects and blasts form T-ALL patients, after $24 \mathrm{~h}$ treatment with AG-490 $(50 \mu \mathrm{M})$ or TRAIL $(25 \mathrm{ng} / \mathrm{ml})$ or the combination of both of the agonists in vitro. Samples treated by AG-490 were always pre-treated for $12 \mathrm{~h}$. Dot plots simultaneously compare the surface levels of phosphatydylserine staining (annexin-V-FITC) and propidium iodide $(\mathrm{PI})$ incorporation. Data are representative of three separate experiments. (B) Flow cytometry analysis of apoptosis of blasts from five T-ALL patients after the treatment with AG$490(50 \mu \mathrm{M})$ or TRAIL $(25 \mathrm{ng} / \mathrm{ml})$ or with both of the agonists combined together in vitro. Samples treated by AG-490 were always pre-treated for $12 \mathrm{~h}$. The combination of both compounds always induced an increase in the cytotoxicity with respect to treatment with TRAIL alone, which was statistically significant $(P$ $<0.01$ ) for four out of five patients. Data are the means of one experiment in triplicate $\pm \mathrm{SD}$. $*(P<0.01)$ indicates statistically significant values with respect to treatment with TRAIL alone. (C) Case study. Age, sex, surface antigen expression and blast density characterizing ALL-T patients. 


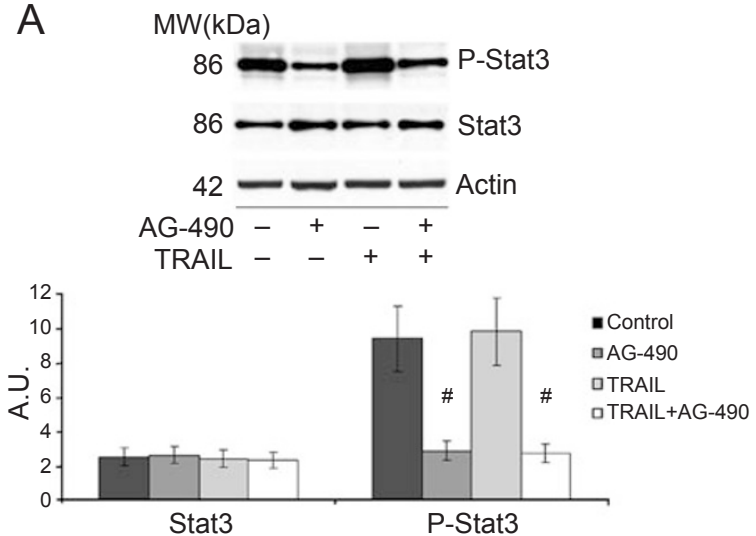

B

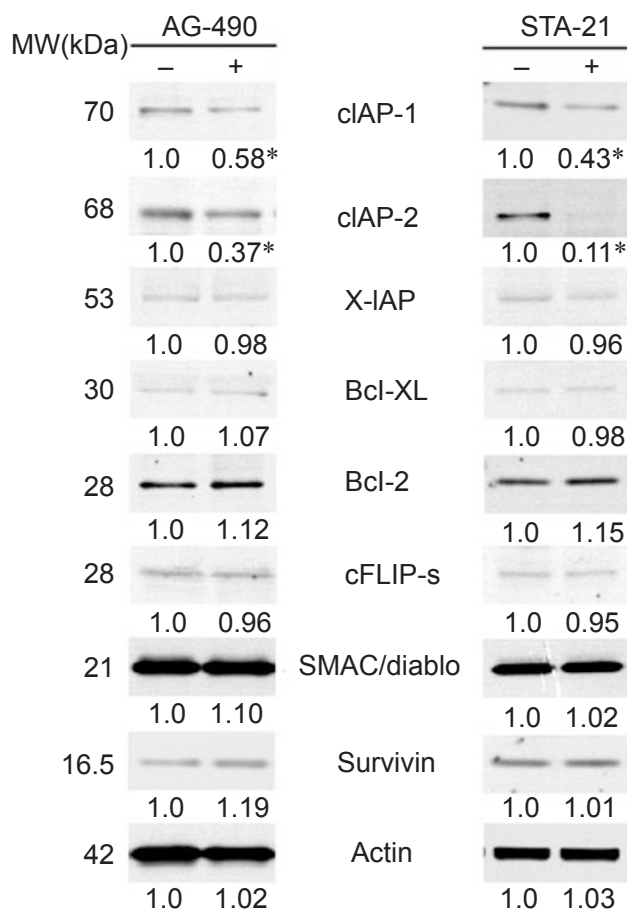

Figure 3 Analysis of Stat3 activation and expression of several apoptosis-related proteins in Jurkat cells treated with AG-490 or STA-21. (A) The intensity of immunoreactivity bands $(10 \mu \mathrm{g}$ of protein) of Stat3 and P-Stat3 was measured by densitometry analysis and expressed as arbitrary units and normalized with respect to the corresponding $\beta$-actin bands (bottom histograms) in Jurkat cells after $24 \mathrm{~h}$ of treatment with AG-490 $(50 \mu \mathrm{M})$ or TRAIL $(25 \mathrm{ng} / \mathrm{ml})$ or the combination of both agonists. Samples treated by AG-490 were always pre-treated for $12 \mathrm{~h}$. $\beta$-actin bands were used as internal control. Data are representative of three separate experiments. \# $(P<0.01)$ indicates statistically significant values with respect to control. (B) Jurkat cells were incubated for $12 \mathrm{~h}$ with AG-490 $(50 \mu \mathrm{M})$ or STA-21 $(30 \mu \mathrm{M})$ or their vehicle $(0.1 \% \mathrm{DMSO})$ alone as control, and the expression of clAP-1, clAP-2, X-IAP, Bcl-XL, Bcl-2, cFLIP-s, SMAC/Diablo, Survivin and Actin was analyzed by western blotting by using the appropriate antibodies. Data are representative of four separate experiments. $\beta$-actin bands were used as internal control. $*(P<0.01)$ indicates statistically significant values with respect to control. in Jurkat cells and whether this effect could modulate the expression of some of the well-known apoptosis inhibitors. Figure 3A shows that, while Stat3 was unaffected, P-Stat 3 was significantly $(P<0.01)$ downregulated with respect to controls and TRAIL alone-treated samples. Such a downregulation was paralleled by an evident decrease of cIAP-1 and cIAP-2 protein expression (Figure 3B, left panel). Similar results were achieved using STA21 (Figure 3B, right panel), a novel Stat3-selective inhibitor [29] that is able to bind SH2 domain of Stat3 and block the dimerization of Stat3 and its DNA binding as well as Stat3-dependent luciferase reporter activity [30, 31].

Flow cytometric analysis of apoptosis confirmed that Stat3 inhibition leads to a significant increase of TRAIL cytotoxicity, giving further strength to the hypothesis of the involvement of Jak2/Stat3 signaling in the modulation of cIAP-1 and cIAP-2 expression (Supplementary information, Figure S2). The analysis of cIAP-1 and cIAP-2 mRNA and protein levels is summarized in Figure $4 \mathrm{~A}$ and $4 \mathrm{~B}$, respectively, showing a significant $(P<0.01)$ decrease of both cIAP-1 and cIAP-2 in AG490 -treated samples. The downregulation of cIAP-1 and cIAP-2 observed upon the addition of AG-490 was maximal at $48 \mathrm{~h}$ of treatment. It is worth noting that no significant modulation of cFLIPs/1 mRNA and protein expression was found in the above reported experiments (Supplementary information, Figure S3). These results supported therefore the hypothesis that P-Stat 3 downregulation and inhibition of cIAP-1 and cIAP-2 expression might be the molecular events involved in the increase of TRAIL-mediated cytotoxicity when it is used in combination with AG-490 (Supplementary information, Figure S4).

cIAP-1 and cIAP-2 knockdown increases TRAIL cytotoxic effects

In order to investigate the possibility that reduction of cIAP-1 and cIAP-2 expression might be responsible for the increased TRAIL sensitivity, we performed RNAimediated knockdown using si-cIAP-1 and/or si-cIAP-2. First, we verified the efficiency of transfection and the effectiveness of silencing. After $24 \mathrm{~h}$, the percentage of transfected cells was about $80 \%$ of living cells. The efficacy of si-cIAP-1 and/or si-cIAP-2 in inhibiting cIAP-1 and cIAP-2 mRNA and protein expression is reported in Figure $5 \mathrm{~A}$ and $5 \mathrm{~B}$. Then, we sought to determine the cytotoxicity of TRAIL in transfected cells. Transfection of si-cIAP-1 and/or si-cIAP-2 in the absence of TRAIL did not lead to significant cell apoptosis (not shown). However, addition of TRAIL in cIAP-1 or cIAP-2 knockdown cells amplified TRAIL-induced cytotoxicity, and the maximum effect was observed when both small interference RNAs (siRNAs) were co-transfected (Figure 5C). 
A
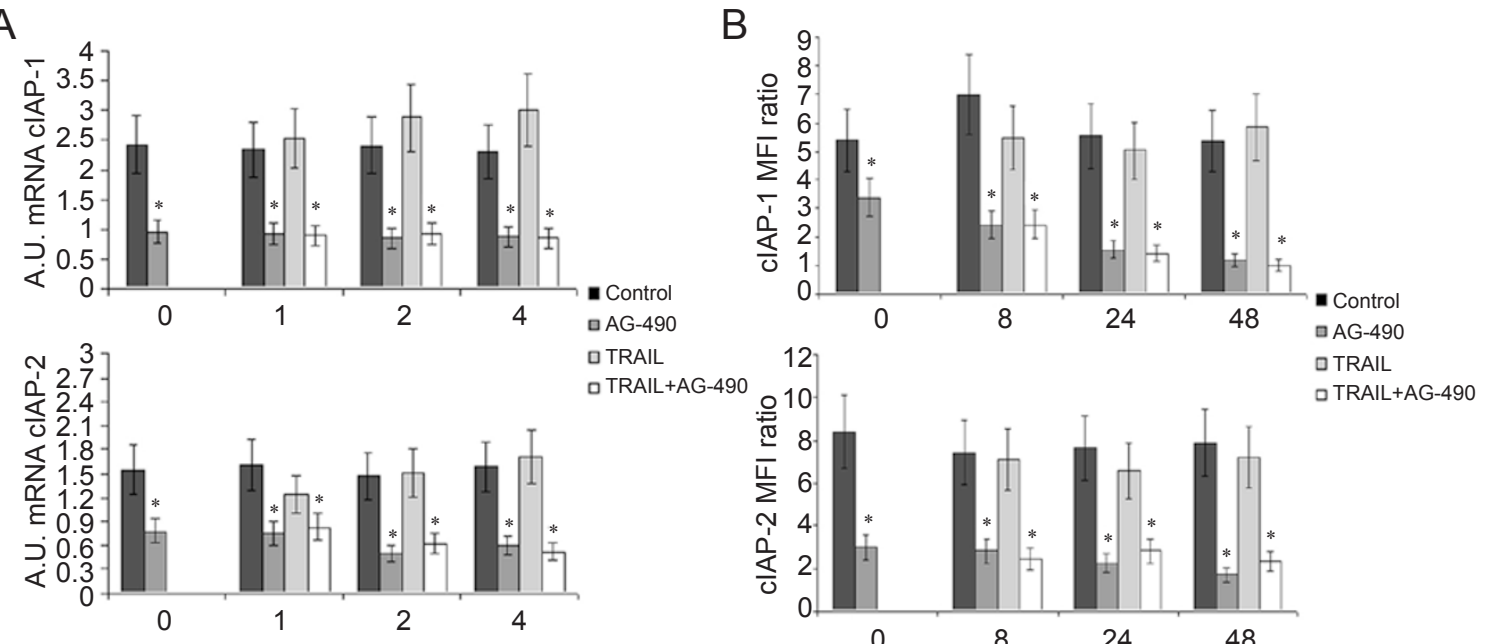

TRAIL

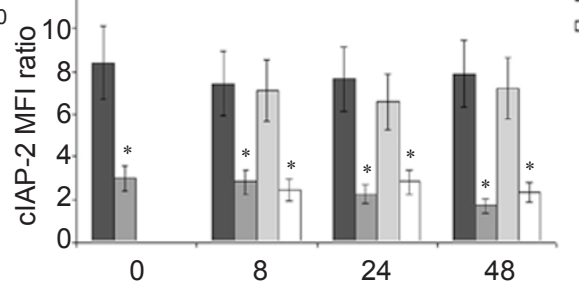

Figure 4 clAP-1 and clAP-2 mRNA transcription and protein levels. (A) clAP-1 and clAP-2 mRNA transcription levels at different time points (0-1-2-4 h) in Jurkat cells treated by AG-490 or TRAIL ( $25 \mathrm{ng} / \mathrm{ml})$ or the combination of both agonists. The intensity of clAP-1 and ClAP-2 and bands was measured, expressed as arbitrary units (A.U.) and normalized to the corresponding GAPDH bands (internal control). Data are the mean of three separate experiments \pm SD. (B) Flow cytometry analysis of intracellular clAP-1 and clAP-2 protein expression levels at different time points (0-8-24-48 h) in Jurkat cells treated by AG-490 or TRAIL $(25 \mathrm{ng} / \mathrm{ml})$ or the combination of both agonists. The expression of clAP-1 and clAP-2 was measured and expressed as mean fluorescence intensity (MFI) ratio. Data are the mean of three separate experiments \pm SD. $*(P<0.01)$ indicates statistically significant values with respect to control samples. In this figure, all samples treated by AG-490 were pretreated for $12 \mathrm{~h}$.

\section{Discussion}

Important breakthroughs in leukemia therapy include clinical applications of agonistic antibodies and small inhibitory molecules $[26,32,33]$. Potential therapeutic effects have also been recently reported for physiological pro-apoptotic proteins such as galectin-1 or TRAIL [34]. Although nowadays most children with T-ALL are cured [35], the outcome for adults with leukemia remains less promising, since adults with T-ALL frequently relapse after conventional therapies [36]. Thus, the development of drug resistance remains a looming problem. One goal is to create therapeutic strategies that minimize chemotherapy doses, without impairing the specific apoptotic effect on tumor cells; therefore, limiting the development of drug-resistance. Apart from the use of the above reported compounds as mono therapy, combinations of traditional chemotherapeutics are particularly interesting in the light of possible enhancement of the effect of mono therapies. In this study, we have demonstrated that in human T cell lines and in T-ALL primary cells, the combination of AG-490 and TRAIL results in a synergistic apoptotic response without inducing toxic effects in normal cells. It has been reported that TRAIL can mediate the biological activity of chemotherapeutic agents [37] and the use of TRAIL as anticancer treatment in vivo has been proposed on the basis of a preferential sensitivity of cancer cells rather than normal cells to TRAIL-induced apoptosis. Nevertheless, the problem of TRAIL-resistance development and some degree of cytotoxicity on normal tissues and cells including hematopoietic cells [38, 39] remain a major issue. In this context, our results indicating that, when combined with AG-490, TRAIL doses could be significantly reduced without affecting, or even enhancing, its cytotoxic effects on cancer cells, provide a new clue that deserves further investigations. Indeed, the possibility of administrating lower doses with very minimal or no toxic effects on normal cells could open new therapeutic perspectives.

We have also addressed the mechanism underlying the synergistic effect of AG-490 and TRAIL on Jurkat cells. Other investigations have highlighted that the combination of drugs such as proteasome inhibitors [40] or ionizing radiation [41] with TRAIL can enhance the apoptotic effect of the latter by modulating the surface expression of TRAIL-R1 and TRAIL-R2 and/or decreasing the levels of the anti-apoptotic protein c-FLIP. In this respect, the lack of influence on the pattern of surface expression of TRAIL receptors following the treatment with AG490 , along with the finding of no consistent modulation of c-FLIP and other apoptosis-related molecules, strongly suggests that there are other possible pathways 
A

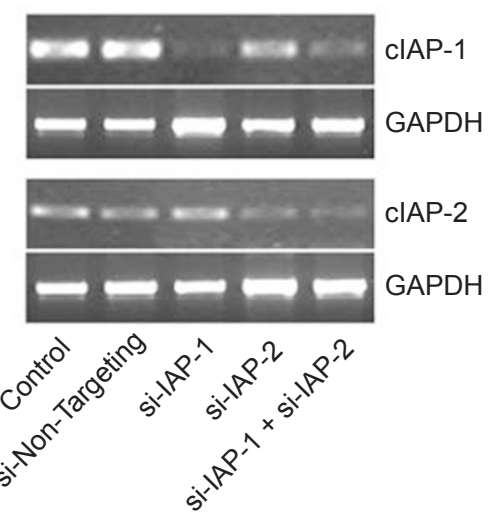

B

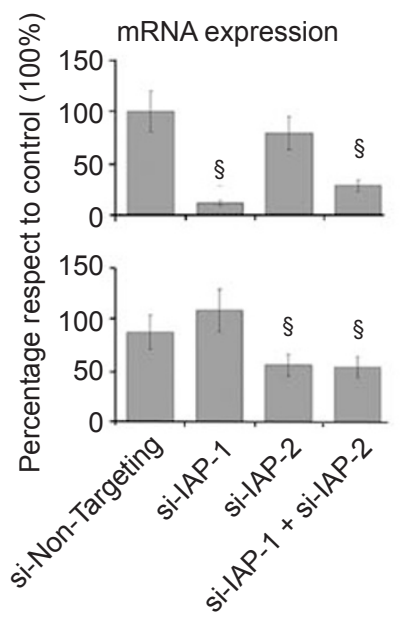

CIAP-2
$\mathrm{MW}(\mathrm{kDa})$

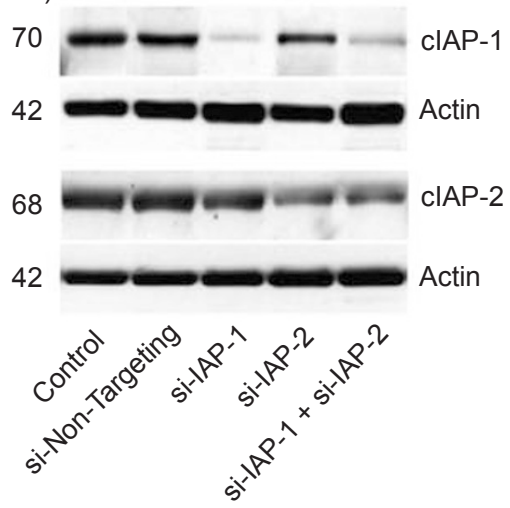

Protein expression
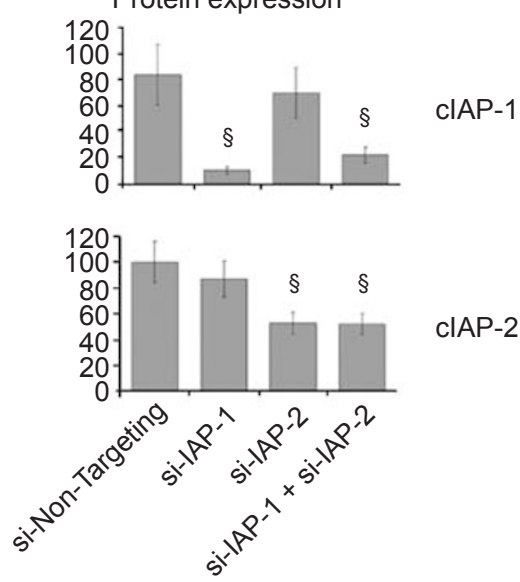

clAP-2

C

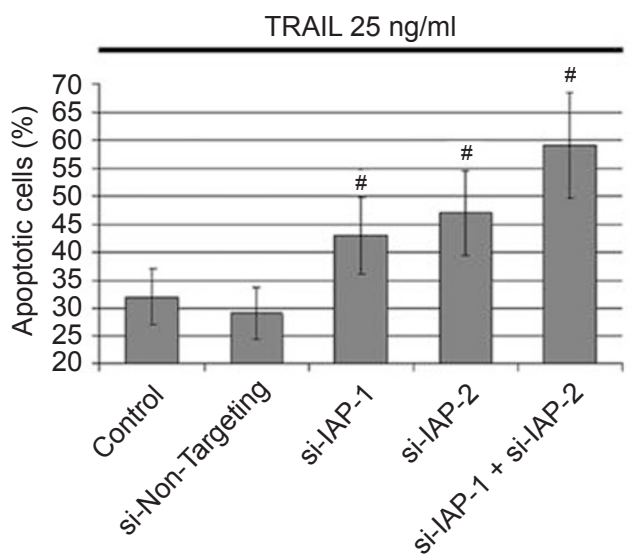

Figure 5 Post-transcriptional gene silencing in Jurkat cells after si-clAP-1 and/or si-clAP-2 nucleofection. (A) mRNA transcription and protein expression levels as evaluated $24 \mathrm{~h}$ after the nucleofection of clAP-1 and clAP-2 in Jurkat cells. GAPDH bands were used as internal control for clAP-1 and clAP-2 mRNA transcription levels, while $\beta$-actin bands were used as internal control for clAP-1 and clAP-2 protein expression levels. Data are representative of three separate experiments. (B) Densitometric analysis of mRNA transcription and protein expression levels of clAP-1 and clAP-2 in Jurkat cells after $24 \mathrm{~h}$ of nucleofection with si-Non-Targeting, si-clAP-1, si-clAP-2 and both silencers combined together. The values, expressed as arbitrary units, have been normalized with respect to GAPDH or to $\beta$-actin bands for mRNA transcription and protein levels, respectively. Bars represent the percentage with respect to control $(100 \%)$. Data are the mean of three separate experiments $\pm \mathrm{SD} . \S(P<0.01)$ indicates statistically significant values with respect to control. (C) Flow cytometry analysis of apoptosis in Jurkat cells induced by TRAIL $(25 \mathrm{ng} / \mathrm{ml})$ for $24 \mathrm{~h}$, after 1 day of si-clAP-1 and/or si-clAP-2 nucleofection. Bars represent the percentage of apoptotic cells evaluated by surface phosphatydylserine staining. Data are the mean of three separate experiments $\pm \mathrm{SD}$. \# $(P<0.01)$ indicates statistically significant values with respect to control. 
responsible for the synergistic apoptotic activity. AG490 is a member of the tyrphostin family of tyrosine kinase inhibitors able to block Jak2/Stat 3 activation in T cells [12]. Of interest, while many kinase inhibitors are often promiscuous in the enzymes they target, AG-490 is unique in that it does not inhibit other lymphocytic tyrosine kinases such as Lyn, Btk, Lck, Sik, Src, Jak1 or Tyk2 [9-12]. The IAP family is a relatively new group of apoptosis-regulating proteins, which consists of a number of proteins that can bind to and inhibit caspases [24]. There is growing evidence that these proteins are expressed in various types of cells including hematopoietic cells and different cancers [42]. It has been demonstrated that the Jak2/Stat3 pathway is constitutively active in $\mathrm{T}$ cell leukemia [43, 44] and that its activation upregulates the expression of IAPs [24], thus promoting the antiapoptotic action [45]. Therefore, inhibition of the Jak2/ Stat3 pathway by the addition of AG-490 to TRAILtreated $\mathrm{T}$ leukemia cells and the consequent downregulation of cIAP-1 and cIAP-2 appear to contribute to the increase of TRAIL apoptotic effect. The use of STA-21, a novel Stat3-selective inhibitor [30], strongly supports the hypothesis that Jak2/Stat3 signaling is responsible for the expression of cIAP-1 and cIAP-2. Of note, cIAP-1 and cIAP-2 have been reported to be overexpressed in $\mathrm{T}$ leukemia cells [46-48]. Their involvement in the signal machinery targeted by the combination of AG-490 and TRAIL (Supplementary information, Figure S4) is suggested by the knockdown experiments performed in TRAIL-treated cells, as the results evidently indicated a relationship between the downregulation of these proteins and the increase of TRAIL-induced cytotoxicity.

The individual different responses of the five ALL patients to the combination of the two compounds remain, anyway, to be explained. Apparently, the efficacy of the combinatorial treatment was inversely related to the extent of the cytotoxic effect when TRAIL was used alone. This observation might be related to the discrete expression of cIAP-1 and cIAP-2 following the treatment with TRAIL alone. It has been suggested that in some cell lines the expression of IAP family proteins might be responsible for the development of the resistance to TRAIL [49]. Therefore, it is possible that different individual expression of cIAP-1 and cIAP-2 in ALL primary cells may differently antagonize the cytotoxicity of TRAIL alone, producing, in turn, a different response upon the addition of AG-490. Taken together, these findings shed light on a novel biological effect produced by the chemotherapeutic drug AG-490, which is able to selectively sensitize T-ALL primary cells and T leukemia cell lines, but not normal T lymphocytes, to TRAIL-induced apoptosis, thus possibly providing new perspectives for the characterization of combined therapies for adult ALLs.

\section{Materials and Methods}

\section{Antibodies}

Anti-TRAIL-R1, anti-TRAIL-R2, anti-TRAIL-R3 and antiTRAIL-R4 antibodies were obtained from R\&D Systems (Minneapolis, MN, USA). Anti-cIAP-1, anti-cIAP-2, anti-FLIP sll $_{\text {, }}$ anti$\mathrm{Bcl}-2$, anti-Bcl-XL, survivin and anti- $\beta$-actin antibodies were obtained from Santa Cruz Biotechnology (Santa Cruz, CA, USA). Anti-Stat3, anti-Phospho-Stat3 (Tyr705), anti-XIAP and antiSMAC/diablo were obtained from Cell Signaling Technology (Danvers, MA, USA). Secondary appropriate Fluorescein Isothiocyanate (FITC)-conjugated antibodies were obtained from Jackson Immunoresearch Laboratories (West Grove, PA, USA), while Horseradish Peroxidase (HRP)-conjugated secondary antibodies were obtained from Santa Cruz Biotechnology.

\section{Cells and human subjects}

Jurkat and SUPT1 cell lines (human leukemia T cells) were purchased from the American Type Culture Collection (Rockville, MD, USA). Subjects with T-ALL were recruited from the Haematology Unit, Pescara hospital, Italy. Five male subjects (aged 36-52) with blast density between 71 000-158 000 white blood cells $/ \mu \mathrm{l}(\mathrm{WBC} / \mu \mathrm{l})$ were characterized as T-ALL patients by using a surface antigen panel, including the characterization of CD14, CD45, CD1a, CD2, CD3, CD4, CD5, CD7, CD8, CD10, CD19, CD22, CD20, HLA-DR, TCR $\alpha / \beta$, TCR $\gamma / \delta, C D 13, C D 11 b, C D 33$, CD34, CD15, CD117, CD61, CD103, TdT, CD79a, MPO (Beckman Coulter, Fullerton, CA, USA). Results of diagnosis exams were reported in Figure 2C. Peripheral blood from ALL-T patients and healthy individuals was collected according to guidelines established by the local ethical committee for human subject studies.

\section{Cell separation}

PBMC were separated from freshly drawn heparinized whole blood by centrifugation ( $400 \times g$ for $30 \mathrm{~min}$ ) over Ficoll-Hypaque (Amersham-Pharmacia Biotec, Uppsala, Sweden).

\section{Cell culture}

Jurkat and SUPT1 cell lines were grown in RPMI-1640 medium (Gibco, Carlsbad, CA, USA) supplemented with $100 \mathrm{U} / \mathrm{ml}$ penicillin, $0.1 \mathrm{mg} / \mathrm{ml}$ streptomycin (Invitrogen, Carlsbad, CA, USA) and $10 \%$ fetal bovine serum (Gibco), at $37{ }^{\circ} \mathrm{C}$ in an atmosphere of $5 \% \mathrm{CO}_{2}$ in air. When required, cells were pre-treated for $12 \mathrm{~h}$ and, then treated with tyrphostin AG-490 (Calbiochem, San Diego, $\mathrm{CA}$, USA) at a final concentration of $50 \mu \mathrm{M}$ [13] for 24 or $48 \mathrm{~h}$, or with different concentrations $(5-300 \mathrm{ng} / \mathrm{ml}$ ) of TRAIL (Alexix Biochemical, San Diego, CA, USA) in the absence or presence of $50 \mu \mathrm{M}$ AG-490, which was added $12 \mathrm{~h}$ before TRAIL. Cell growth and viability were quantified by trypan blue exclusion test. When required, cells were treated for 24 and $48 \mathrm{~h}$ with a Stat3 highly selective inhibitor (STA-21 $30 \mu \mathrm{M})$ (Biomol International, Plymouth Meeting, PA, USA) [30, 31] alone, or with $25 \mathrm{ng} / \mathrm{ml}$ of TRAIL alone or with the combination of both the molecules. Treatments with STA-21 always started $12 \mathrm{~h}$ before the onset of the experiments.

\section{MTT assay}

A total of $5 \times 10^{5}$ cells stimulated with $50 \mu \mathrm{M} \mathrm{AG}-490,25 \mathrm{ng} / \mathrm{ml}$ TRAIL, or both for 24 and $48 \mathrm{~h}$ were incubated with $5 \mathrm{mg} / \mathrm{ml}$ MTT (Sigma-Aldrich, St Louis, MO, USA) for $3 \mathrm{~h}$ at $37^{\circ} \mathrm{C}$ in trip- 
licate. After a wash, cells were solubilized with DMSO (SigmaAldrich) and quantified by measuring the absorbance at $570 \mathrm{~nm}$ in a micro plate reader Spectra Max 190 (Molecular Devices, Sunnyvale, CA, USA). Data analysis was performed with a SOFTmax PRO 4.0 software (Molecular Devices).

\section{Reverse transcriptase-PCR analysis}

Total RNA was isolated from Jurkat cells with SV Total RNA Isolation System (Promega Corporation, Madison, WI, USA). To generate cDNA, $1 \mu \mathrm{g}$ of total RNA in reaction mixture with Oligo (dT) primer and MMLV reverse transcriptase $(100 \mathrm{U} / \mu \mathrm{l})($ Celbio, Milan, Italy), was used. Semi quantitative RT-PCR analysis was performed using PCR system 9700 (Applied Biosystems, Foster City, CA, USA). PCR reaction mixture contained cDNA, dNTPs $(10 \mathrm{mM})$, Taq polymerase $(5 \mathrm{U} / \mu \mathrm{l})$ (Biotools B\&M Labs, Madrid, Spain) and $\mathrm{MgCl}_{2}(1.5 \mathrm{mM})$. The conditions for PCR amplification were as follows: $2 \mathrm{~min}$ at $95^{\circ} \mathrm{C}$ for one cycle, 30 cycles of $30 \mathrm{~s}$ at $95{ }^{\circ} \mathrm{C}$ followed by $30 \mathrm{~s}$ at $56{ }^{\circ} \mathrm{C}$ and $30 \mathrm{~s}$ at $72{ }^{\circ} \mathrm{C}$ for cIAP- 1 and cIAP-2 primers; 2 min at $95^{\circ} \mathrm{C}$ for one cycle, 30 cycles of $45 \mathrm{~s}$ at $95{ }^{\circ} \mathrm{C}$ followed by $45 \mathrm{~s}$ at $55^{\circ} \mathrm{C}$ and $45 \mathrm{~s}$ at $72{ }^{\circ} \mathrm{C}$ for cFLIPs/ 1 primers. cDNA was amplified using the following primers: cIAP-1 5'GCT GTT GTC AAC TTC AGA TAC CAC T-3' (sense), 5'-TGT TTC ACC AGG TCT CTA TTA AAG CC-3' (antisense); cIAP-2 5'-ACT TGA ACA GCT GCT ATC CAC ATC-3' (sense), 5'-GTT GCT AGG ATT TTT CTC TGA ACT GTC-3' (antisense); cFLIPs/ 1 5'-TGT TGC TAT AGA TGT GG-3' (sense), 5'-AAG GAT CCT TGA GAC TCT-3' (antisense) [50-52]. As internal control, GAPDH amplified from sense and antisense primers (5'-GAA GGT GAA GGT CGG AGT CA-3' and 5'-TTC ACA CCC ATG ACG AAC AT- $3^{\prime}$ ) was used. The PCR products were run on a $2 \%$ agarose gel containing ethidium bromide and visualized by Gel Doc (Bio Rad, Hercules, CA, USA). Densitometry analysis of PCR products was performed using Quantity ONE 4.3.1 (Bio Rad).

\section{Western blotting}

A total of $1 \times 10^{6}$ cells were harvested by centrifugation and solubilized in a lysis buffer containing $50 \mathrm{mM}$ Tris-HCl $(\mathrm{pH} 7.4)$, $150 \mathrm{mM} \mathrm{NaCl}, 1 \%$ IGEPAL CA-630 (Sigma-Aldrich), $0.1 \%$ sodium dodecyl sulfate (Sigma-Aldrich), $0.5 \%$ deoxycholic acid sodium salt added with $1 \mathrm{mM} \mathrm{Na} \mathrm{VO}_{4}, 1 \mu \mathrm{g} / \mathrm{ml}$ leupeptin, $1 \mu \mathrm{g} / \mathrm{ml}$ aprotinin and $0.5 \mathrm{mM}$ phenyl-methanesulfonyl fluoride. Total lysates (10 $\mu \mathrm{g}$ of protein) were separated on NuPAGE $4-12 \%$ Bis-Tris Gel (Invitrogen) and blotted onto nitrocellulose membrane (Amersham). After saturation with 5\% skim milk powder (Sigma-Aldrich) in tris buffer saline pH 7.4 and $0.05 \%$ tween20 (Sigma-Aldrich), the membranes were incubated with $0.2 \mu \mathrm{g} / \mathrm{ml}$ anti-cIAP-1, anticIAP-2, anti-FLIP $\mathrm{s}_{\mathrm{s} /}$, anti-Bcl-2, anti-Bcl-XL, survivin, anti-Stat3, anti-Phospho-Stat3 (Tyr705), anti-XIAP and anti-SMAC/diablo and anti- $\beta$-actin (internal control) antibodies followed by the appropriate HRP-conjugated secondary antibodies $(0.27 \mu \mathrm{g} / \mathrm{ml})$. Immunoreactive bands were detected by using the ECL system (Santa Cruz Biotechnology), according to the manufacturer's instructions. Chemiluminescence was detected by Gel Doc (Bio Rad) and analyzed by Quantity ONE 4.3.1 (Bio Rad) [53].

\section{Detection of apoptosis by flow cytometry}

Human annexin V-FITC kit (Bender MedSystems, Vienna, Austria) was used to detect phosphatidylserine expression on cell membranes, in order to discriminate apoptotic cells, counterstained by PI. Each sample $\left(5 \times 10^{5}\right.$ cells $)$ was treated according to the manufacturer's instructions. After staining procedures, samples were analyzed by flow cytometry using a FACSCalibur cytometer (Becton Dickinson, Franklin Lakes, NJ, USA). Finally, data were analyzed using CELLQuest 3.2.1.f1 software (Becton Dickinson).

\section{Analysis of TRAIL receptor expression by flow cytometry}

For each staining $5 \times 10^{5}$ cells were incubated with $20 \mu \mathrm{g} / \mathrm{ml}$ antiTRAIL-R1, anti-TRAIL-R2, anti-TRAIL-R3 or anti-TRAIL-R4 in PBS containing $0.5 \%$ albumin from bovine serum (BSA, SigmaAldrich) for $30 \mathrm{~min}$, followed by $15 \mu \mathrm{g} / \mathrm{ml} \mathrm{FITC-conjugated} \mathrm{ap-}$ propriate secondary antibody in PBS containing $0.5 \%$ BSA for 30 min. Secondary antibody-matched controls were used to assess unspecific fluorescence in the FL-1 channel. After staining procedures, samples were analyzed by flow cytometry using a FACSCalibur cytometer (Becton Dickinson). Finally, data were analyzed using CELLQuest 3.2.1.f1 software (Becton Dickinson).

Flow cytometry analysis of intracellular cIAP-1 and CIAP-2

A total of $5 \times 10^{5}$ cells were fixed with $2 \%$ paraformaldehyde for $5 \mathrm{~min}$ at $25^{\circ} \mathrm{C}$ and treated with $150 \mathrm{mM} \mathrm{NaCl}, 5 \mathrm{mM}$ EDTA, $50 \mathrm{mM}$ Tris- $\mathrm{HCl} \mathrm{pH} 7.4,0.05 \%$ IGEPAL CA-630 (Sigma-Aldrich), $0.25 \%$ lambda-carrageenan (Sigma-Aldrich) and $0.02 \% \mathrm{NaN}_{3}$ (Sigma-Aldrich) (NET Gel). For each staining, $2 \mu \mathrm{g} / \mathrm{ml}$ anticIAP-1 or anti-cIAP-2 antibody in NET Gel, was reacted with the sample for $30 \mathrm{~min}$, followed by incubation with $15 \mu \mathrm{g} / \mathrm{ml} \mathrm{FITC-}$ conjugated appropriate secondary antibodies in NET Gel for 30 min. Secondary antibody-matched controls were used to assess unspecific fluorescence in FL-1. After staining procedures, samples were analyzed by flow cytometry using a FACSCalibur cytometer (Becton Dickinson). Finally, data were analyzed using CELLQuest 3.2.1.f1 software (Becton Dickinson).

\section{Nucleofection}

All transient transfections were performed by using a nucleofector device associated to its corresponding kits (Amaxa, Inc., Cologne, Germany). A total of $3 \times 10^{6}$ Jurkat cells per sample were resuspended in $100 \mu \mathrm{l}$ of the nucleofector solution containing $100 \mathrm{nM}$ siRNA and nucleofected according to Amaxa's protocol. Cells were seeded at a density of $1.5 \times 10^{5}$ per coverslip in a 24 well plate. First, the transfection program for Jurkat cells was optimized. Briefly, $3 \times 10^{6}$ Jurkat cells were transfected with the nucleofector solution $\mathrm{V}$, using different nucleofector programs (C-16, I-10 and S-18); the I-10 program provided the highest transfection efficiency associated with the lowest cell mortality and was identified as the best program (90\% live cells). A Green Fluorescent Protein (GFP) expression vector pEGFP-C1 (Clonotech, Mountain, California, USA) was co-transfected with the respective GFP siRNA sequences. After $24 \mathrm{~h}$, a specific flow cytometric analysis indicated that the percentage of GFP-positive cells was around $80 \%$ of the living cells. Successively, Jurkat cells were nucleofected with the following siRNA: non-targeting siRNA pool, siGENOME SMART pool BIRC2 (cIAP-1) and siGENOME SMART pool BIRC3 (cIAP-2) (Dharmacon, Lafayette, CO, USA).

\section{Statistical analysis}

Data were analyzed by ANOVA and Mann-Whitney rank-sum tests. Data are shown as mean \pm SD unless otherwise specified. Statistical significance was defined as $P<0.01$. 


\section{Acknowledgments}

This work was supported by Italian MIUR-COFIN 2007 funds, by Fondazione Carichieti, Chieti, Italy and by a generous donation of Marco Romanelli and Donatella De Sanctis on the day of their wedding. The authors wish to thank Prof John C Byrd (The Ohio State University, USA) for his critical review of the manuscript.

\section{References}

1 Eden T, Pui CH, Schrappe M, Tognoni G, Masera G. All children have a right to full access to treatment for cancer. Lancet 2004; 364:1121-1122.

2 Kaufman SH, Steensma DP. On the TRAIL of a new therapy for leukemia. Leukemia 2005; 19:2195-2202.

3 Younes A, Kadin ME. Emerging applications of the tumor necrosis factor family of ligands and receptors in cancer therapy. J Clin Invest 2003; 21:3526-3534.

4 Kelley SK, Ashkenazi A. Death to the bad guys: targeting death receptors in cancer with Apo2L/TRAIL. Curr Opin Pharmacol 2004; 4:333-339.

5 Duiker EW, Mom $\mathrm{CH}$, de Jong $\mathrm{S}$, et al. The clinical trail of TRAIL. Eur J Cancer 2006; 42:2233-2240.

6 MacFarlane M. TRAIL-induced signalling and apoptosis. Toxicol Lett 2003; 139:89-97.

7 Johnstone RW. Histone-deacetylase inhibitors: novel drugs for the treatment of cancer. Nat Rev Drug Discov 2002; 1:287-299.

8 Singh TR, Shankar S, Srivastava RK. HDAC inhibitors enhance the apoptosis-inducing potential of TRAIL in breast carcinoma. Oncogene 2005; 24:4609-4623.

9 Burdelya L, Catlett-Falcone R, Levitzki A, et al. Combination therapy with AG-490 and interleukin 12 achieves greater antitumor effects than either agent alone. Mol Cancer Ther 2002; 1:893-899.

10 Rahaman SO, Harbor PC, Chernova O, Barnett GH, Vogelbaum MA, Haque SJ. Inhibition of constitutively active Stat3 suppresses proliferation and induces apoptosis in glioblastoma multiforme cells. Oncogene 2002; 21:8404-8413.

11 Toyonaga T, Nakano K, Nagano M, et al. Blockade of constitutively activated Janus kinase/signal transducer and activator of transcription-3 pathway inhibits growth of human pancreatic cancer. Cancer Lett 2003; 201:107-116.

12 Wang LH, Kirken RA, Erwin RA, Yu CR, Farrar WL. JAK3, STAT, and MAPK signaling pathways as novel molecular targets for the tyrphostin AG-490 regulation of IL-2-mediated T cell response. J Immunol 1999; 162:3897-3904.

$13 \mathrm{Yu}$ JH, Kim KH, Kim H. Suppression of IL-1 $\beta$ expression by the Jak2 inhibitor AG-490 in cerulean-stimulated pancreatic acinar cells. Biochem Pharmacol 2006; 72:1555-1562.

14 De Vos J, Jourdan M, Tarte K, Jasmin C, Klein B. JAK2 tyrosine kinase inhibitor tyrphostin AG-490 down regulates the mitogen activated protein kinase (MAPK) and signal transducer and activator of transcription (STAT) pathways and induces apoptosis in myeloma cells. Br J Haematol 2000; 109:823-828.

15 Takemoto S, Mulloy JC, Cereseto A, et al. Proliferation of adult $\mathrm{T}$ cell leukemia/lymphoma cells is associated with the constitutive activation of JAK/STAT proteins. Proc Natl Acad Sci USA 1997; 94:13897-13902.
16 Stepkowski SM and Kirken RA. Unique advantages of Janus Kinase 3 as a target for selective and nontoxic immunosuppression. Expert Rev Clin Immunol 2005; 1:307-310.

17 Meydan N, Grunberger T, Dadi H, et al. Inhibition of acute lymphoblastic leukaemia by a Jak-2 inhibitor. Nature 1996; 379:645-648.

18 Kirken RA, Erwin RA, Taub D, et al. Tyrphostin AG-490 inhibits cytokine-mediated JAK3/STAT5a/b signal transduction and cellular proliferation of antigen-activated human $\mathrm{T}$ cells. J Leukoc Biol 1999; 65:891-899.

19 Yeh TC, Pellegrini S. The Janus Kinase family of protein tyrosine kinase and their role in signalling. Cell Mol Life Sci 1999; 19:1523-1534.

20 Mazurkiewicz-Munoz AM, Argetsinger LS, Kouadio JL, et al. Phosphorylation of JAK2 at serine 523: a negative regulator of JAK2 that is stimulated by growth hormone and epidermal growth factor. Mol Cell Biol 2006; 26:4052-4062.

21 Stephanou A, Brar BK, Knight RA, Latchman DS. Opposing actions of STAT-1 and STAT-3 on the Bcl-2 and Bcl-x promoters. Cell Death Differ 2000; 7:329-330.

22 Alas S, Bonavida B. Rituximab inactivates signal transducer and activation of transcription 3 (STAT3) activity in B-nonHodgkin's lymphoma through inhibition of the interleukin 10 autocrine/paracrine loop and results in down-regulation of Bcl-2 and sensitization to cytotoxic drugs. Cancer Res 2001; 61:5137-5144.

23 Alas S, Bonavida B. Inhibition of constitutive STAT3 activity sensitizes resistant non-Hodgkin's lymphoma and multiple myeloma to chemotherapeutic drug-mediated apoptosis. Clin Cancer Res 2003; 9:316-326.

24 Hasegawa T, Suzuki K, Sakamoto C, et al. Expression of the inhibitor of aoptosis (IAP) family members in human neutrophils: up-regulation of cIAP-2 by granulocyte colonystimulating factor and overexpression of cIAP-2 in chronic neutrophilic leukaemia. Blood 2003; 101:1164-1171.

25 Bremer E, van Dam G, Kroesen BJ, de Leij L, Helfrich W. Target induction of apoptosis for cancer therapy: current progress and prospects. Trends Mol Med 2006; 12:382-393.

26 Schimmer AD, Welsh K, Pinilla C, et al. Small-molecule antagonists of apoptosis suppressor XIAP exhibit broad antitumor activity. Cancer Cell 2004; 5: 25-35.

27 Deveraux QL, Reed JC. IAP family proteins-suppressors of apoptosis. Gen Dev 1999; 13:239-252.

28 Chu ZL, McKinsey TA, Liu L, Gentry JJ, Malim MH, Ballard DW. Suppression of tumor necrosis factor-induced cell death

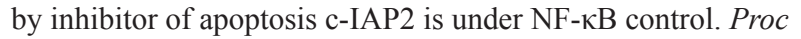
Natl Acad Sci USA 1997; 94:10057-10062.

29 Song H, Wang R, Wang S, Lin J. A low-molecular-weight compound discovered through virtual database screening inhibits Stat3 function in breast cancer cells. Proc Natl Acad Sci USA 2005; 102:4700-4705.

30 Bhasin D, Cisek K, Pandharkar T, et al. Design, synthesis, and studies of small molecule STAT3 inhibitors. Bioorg Med Chem Lett 2008; 18:391-395.

31 Chen CL, Loy A, Cen L, et al. Signal transducer and activator of transcription 3 is involved in cell growth and survival of human rhabdomyosarcoma and osteosarcoma cells. BMC Cancer 2007; 7:78-90.

32 Mori E, Thomas M, Motoki K, Kataika S. Distinct function of 
monoclonal antibody to TRAIL-R2 as potentiator or inhibitor of the ligand TRAIL-induced apoptosis. FEBS Lett 2005; 579:5379-5384.

33 Mori E, Thomas M, Motoki K, et al. Human normal hepatocytes are susceptible to apoptosis signal mediated by both TRAIL-R1 and TRAIL-R2. Cell Death Differ 2004; 11:203207.

34 Mazurek N, Sun YJ, Liu KF, et al. Phosphorylated galectin-3 mediates tumor necrosis factor-related apoptosis-inducing ligand (TRAIL) signaling by regulating PTEN in human breast carcinoma cells. $J$ Biol Chem 2007; 282:21337-21348.

35 Pui CH, Evans WE. Acute lymphoblastic leukemia. $N$ Engl $J$ Med 1998; 339:605-615.

36 Litzow MR. Acute lymphoblastic leukemia in adults. Curr Treat Options Oncol 2000; 1:19-29.

37 Wang P, Song JH, Song DK, Zhang J, Hao C. Role of death receptor and mitochondrial pathways in conventional chemotherapy drug induction of apoptosis. Cell Signal 2006; 18:1528-1535.

38 Mori T, Doi R, Toyoda E, et al. Regulation of the resistance to TRAIL-induced apoptosis as a new strategy for pancreatic cancer. Surgery 2005; 138:71-77.

39 Meurette O, Fontaine A, Rebillard A, et al. Cytotoxicity of TRAIL/anticancer drug combinations in human normal cells. Ann N Y Acad Sci 2006; 1090:209-216.

40 Ganten TM, Koschny R, Haas TL, et al. Proteasome inhibition sensitizes hepatocellular carcinoma cells, but not human hepatocytes, to TRAIL. Hepatology 2005; 42:588-597.

41 Di Pietro R, Secchiero P, Rana R, et al. Ionizing radiation sensitizes erythroleukemic cells but not normal erythroblasts to tumor necrosis factor-related apoptosis-inducing ligand (TRAIL)-mediated cytotoxicity by selective up-regulation of TRAIL-R1. Blood 2001; 97:2596-2603.

42 de Graaf AO, van Krieken JH, Tonnissen E, et al. Expression of C-IAP1, C-IAP2 and SURVIVIN discriminates different types of lymphoid malignancies. Br J Haematol 2005; 130:852-859.

43 Lin TS, Mahajan S, Frank DA. STAT signaling in the pathogenesis and treatment of leukemias. Oncogene 2000; 19:24962504.

44 Rajasingh J, Raikwar HP, Muthian G, Johnson C, Bright JJ. Curcumin induces growth-arrest and apoptosis in association with the inhibition of costitutively active JAK-STAT pathway in T cell leukemia. Bioc Biophis Res Comm 2006; 340:359368 .

45 Tomita M, Kawakami H, Uchihara JN, et al. Inhibition of constitutively active Jak-Stat pathway suppresses cell growth of human T-cell leukemia virus type 1-infected T-cell lines and primary adult T-cell leukemia cells. Retrovirology 2006; 3:22-32.

46 Moriai R, Asanuma K, Kobayashi D, et al. Quantitative analysis of the anti-apoptotic gene surviving expression in malignant haematopoietic cells. Anticancer Res 2001; 21:595600 .

47 Nakagawa Y, Yamaguchi S, Hasegawa M, et al. Differential expression of survivin in bone marrow cells from patients with acute lymphocytic leukaemia and chronic lymphocytic leukemia. Leukemia Res 2004; 28:487-494.

48 Kamihira S, Yamada Y, Hirakata Y, et al. Aberrant expression of caspase cascade regulatory genes in adult T-cell leukaemia: survivin is an important determinant for prognosis. $\mathrm{Br}$ J Haematol 2001; 114:63-69.

49 Mirandola P, Sponzilli I, Gobbi G, et al. Anticancer agents sensitize osteosarcoma cells to TNF-related apoptosis-inducing ligand downmodulating IAP family proteins. Int J Oncol 2006; 28:127-133.

50 Uno K, Inukai T, Kayagaki N, et al. TNF-related apoptosis-inducing ligand (TRAIL) frequently induces apoptosis-inducing ligand (TRAIL) frequently induces apoptosis in Philadelphia chromosome positive leukaemia cells. Blood 2003; 101:36583667.

51 Pitti RM, Marsters SA, Ruppert S, Donahue CJ, Moore A, Ashkenazi A. Induction of apoptosis by Apo-2 ligand, a new member of the tumor necrosis factor cytokine family. $J$ Biol Chem 1996; 271:12687-12690.

52 Chen HX, Liu YJ, Zhou XD, Lou RY. Expression of cellular FLICE/caspase- 8 inhibitory protein is associated with malignant potential in endometrial carcinoma. Int J Gynecol Cancer 2005; 15:663-670.

53 Bertagnolo V, Brugnoli F, Marchisio M, Celeghini C, Carini C, Capitani S. Association of PI 3-K with tyrosine phosphorylated Vav is essential for its activity in neutrophil-like maturation of myeloid cells. Cell Signal 2004; 16:423-433.

(Supplementary information is linked to the online version of the paper on the Cell Research website.) 